\title{
Comparison of channelled vs unchannelled video laryngoscope- A prospective randomised trial
}

\author{
Shibani Padhy ${ }^{1}$, Kavitha Jayaram ${ }^{2, *}$, Sindhuja $^{3}$, Priyanka ${ }^{4}$ \\ ${ }^{\mathbf{1}}$ Assistant Professor, ${ }^{\mathbf{2}}$ Associate Professor, ${ }^{\mathbf{3}, \mathbf{4}}$ Junior Resident, Dept. of Anaesthesiology, Nizam's Institute of Medical Sciences, \\ Hyderabad, Telangana, India \\ *Corresponding Author: Kavitha Jayaram \\ Email: drkarunya@gmail.com
}

Received: $10^{\text {th }}$ April, 2018

Accepted: $28^{\text {th }}$ June, 2018

\begin{abstract}
Introduction: Conventional laryngoscopy using the traditional Macintosh blade remains the commonest method of tracheal intubation in the routine clinical scenario. Video laryngoscopes have taken over this position in many scenarios as they have been proved successful over conventional laryngoscope. There are several types of video laryngoscopes available with multiple advantages over one another. We aimed to compare the performance of the Airtraq and McGrath when performing tracheal intubation in routine anaesthesia practice

Materials and Methods: Sixty patients posted for surgery requiring endotracheal intubation were randomized to undergo intubation using an Airtraq $(n=30)$ or McGrath $(n=30)$ video laryngoscope. Primary end points were total duration of intubation, overall success rate of tracheal intubation, number of optimization manoeuvres used and ease of intubation between the two groups.

Results: We observed that the duration of successful intubation was shorter in Airtraq $19.18( \pm 2.86)$ secs $)$ than in the McGrath laryngoscope. $27( \pm 5.09)$ secs $^{*} \mathrm{P}<0.001$. IDS scores were also better in the Airtraq group; grade 0 (easy) $73.3 \%$ easy versus $36.7 \%$ in the McGrath group, $\mathrm{P}<0.001$. A significantly better Cormack lehane view was obtained with the Airtraq. Grade I in $73.3 \%$ with the Airtraq group versus $50 \%$ in the McGrath group, $\mathrm{p}<0.001$. Airtraq required lesser optimization manoeuvres for successful intubation. There were no differences in the hemodynamic profiles post intubation between the two groups.

Conclusion: We conclude that the Airtraq laryngoscope facilitates a more rapid and successful intubation as compared to McGrath in routine clinical practice.
\end{abstract}

Keywords: Airtraq, McGrath, Intubation.

\section{Introduction}

Conventional direct laryngoscopy with a Macintosh blade remains the standard technique for placing an endotracheal tube during routine airway management. However, unanticipated difficult and failed tracheal intubation are among the major causes of airway related morbidity and mortality in the perioperative period. $^{1}$ In recent years, video laryngoscopes have gained popularity as an important tool in airway management armamentarium. ${ }^{2}$ Video laryngoscopes allow better visualization of the glottis structures without requiring alignment of the oral, pharyngeal and laryngeal axes which is in contrast to conventional laryngoscope. ${ }^{3}$ In addition, they are a great educational tool for training novices in tracheal intubation. With the realization of these advantages, many indirect laryngoscopes with different shapes and imaging technologies have been developed in the recent times.

The McGrath $^{\circledR}$ series 5 video laryngoscope (Aircraft Medical, Edinburgh, Scotland) which incorporates a light source and a camera at the tip of the blade provides better view of the vocal cords and the surrounding anatomical structures on a LCD screen attached to the laryngoscope handle. ${ }^{4}$ The McGrath is positioned in a similar fashion to the conventional
Macintosh blade and the endotracheal tube is advanced through the vocal cords under direct vision.

The Airtraq $^{\circledR}$ incorporates two channels; one transfers the image to a proximal viewfinder via a series of prisms and lenses, while the other works as a conduit for the endotracheal tube. ${ }^{5}$ Airtraq is therefore a channelled video laryngoscope and provides the desired curvature needed for intubation. ${ }^{5}$

Both the devices have demonstrated superiority over a Macintosh laryngoscope in the difficult airway scenario. ${ }^{5,6}$ They provide good optical view of glottis and improve the Cormack lehane grading.

However there is a paucity of studies comparing the intubation characteristics of McGrath and Airtraq laryngoscopes in the normal airway in daily clinical practice. Hence, in this study, we compared the performance of McGrath and Airtraq laryngoscopes using Intubation difficulty score as primary outcome measure. Secondary outcome measures include time to intubation (TTI), number of attempts, glottis view, hemodynamic responses, incidence of airway trauma, and ease of tracheal intubation in routine anaesthesia practice.

\section{Materials and Methods}

After obtaining institutional ethics committee approval and informed consent, American Society of 
Anaesthesiologists Physical Status (ASA) 1 or 2 patients of either sex, between the ages of 18-60 yr undergoing elective surgery requiring endotracheal intubation were considered for the study. Exclusion criteria included, ASA 3 or 4 patients, those at an increased risk of aspiration, difficult intubation (Mallampati class III or IV, retrognathia, restricted neck movements, thyromental distance $<6 \mathrm{~cm}$; inter-incisor distance $<3.5 \mathrm{~cm}$ ) and patients with coagulopathies. All preoperative data like airway parameters, demographic data were collected by an independent unblinded anaesthesiologist.

Patients were randomly allocated to either the Airtraq ${ }^{\circledR}$, or McGrath videolayngoscope group using a computer-generated random code enclosed within sealed envelopes. All patients received a standard monitoring including ECG, non-invasive arterial pressure, pulse oximetry, end-tidal carbon dioxide monitoring and Entropy ${ }^{\circledR}$ monitoring. All patients received a uniform anaesthesia protocol, which included premedication with fentanyl $\left(1.5 \mu \mathrm{g} \mathrm{kg}^{-1}\right)$; induction with i.v. Propofol $\left(2-4 \mathrm{mg} \mathrm{kg}^{-1}\right)$ titrated to loss of verbal response. This was followed by manual ventilation with sevoflurane $(2.0 \%)$ in oxygen, and administration of rocuronium $(1 \mathrm{mg} / \mathrm{kg}$ i.v) After the onset of neuromuscular block, endotracheal intubation was performed in the sniffing position with a pillow under the head by a single anaesthesiologist experienced in the use both the video laryngoscopes. Anaesthesia was maintained with controlled ventilation and with sevoflurane $(1.25-1.75 \%)$ in a mixture of air and oxygen in a 1:1 ratio. No surgical procedure was initiated or additional drug administered, during the 5 min data collection period after tracheal intubation. Subsequent management of the patient was at the discretion of anaesthesiologist caring for the patient.

The primary outcome measure was the intubation difficulty scale (IDS) score. The IDS, as described by Adnet and colleagues, ${ }^{7}$ is a seven-point scoring system which indicates the difficulty of intubation based on parameters like number of attempts, Cormack and Lehane score, lifting force required, and the position of the vocal cords. An IDS of 0 denotes ideal intubating conditions and the score progresses with increasingly difficult tracheal intubations. The IDS was classified as easy for score 0 , slight difficulty for range 0-5, Moderate to Major difficulty if score is $>5$, impossible intubation for infinity. ${ }^{7}$

The duration of the intubation was defined as the time taken from insertion of the blade between the teeth until the TT was placed through the vocal cords, as confirmed by end tidal carbon dioxide monitoring. Other end points included the total number of intubation attempts, number of optimization manoeuvres required (use of a bouggie, external laryngeal pressure, lifting force), the Cormack and Lehane grade $^{8}$ and the total number of passes of the ETT. The optimization manoeuvres required for intubation were rated on a scale of 0 to $2(0-$ No manoeuvres required. 1- External laryngeal pressure. 2Use of bouggie).

An attempt lasting for more than $120 \mathrm{~s}$ or drop in saturation to less than $92 \%$, was stopped and a maximum of three attempts were allowed. More than three attempts, duration of $120 \mathrm{~s}$ and use of alternative laryngoscope were regarded as intubation failures. In the event of intubation failure, conventional difficult intubation protocols were followed.

Ease of intubation with each of the two devices was assessed on a score of 1 to 3: 1. Easy endotracheal intubation without manoeuvre. 2. Satisfactory - tracheal intubation with manoeuvres. 3. Difficult - tracheal intubation not possible even with manoeuvres.

\section{Statistical Analysis}

Sample size determination was based on the IDS score. Based on previous studies we assumed a clinically important change in mean IDS score between groups to be 2.0. ${ }^{9}$ Taking a standard deviation (SD) of 2.25 from prior studies, ${ }^{16}$ and using $\mathrm{a}=0.05$ and a $\beta=0.2$, we estimated that 30 patients would be required per group.

SPSS 17.0 software (Chicago, IL) was used for statistical analyses. Data are presented as means (SD) or as medians (inter-quartile range), as appropriate. Student's $t$ test or Mann-Whitney $U$ test was used for comparison of quantitative data and chi squared test or Fisher's exact test was used for comparison of qualitative data. $P$-value was less than 0.05 was considered statistically significant.

\section{Results}

All 60 patients randomly allocated to Airtraq (group I) or McGrath (group II) completed the study. Both groups were similar with respect to demographic data and baseline airway data [Table 1]. 
Table 1: Demographic variables and baseline airway data

\begin{tabular}{|l|c|c|c|}
\hline \multicolumn{1}{|c|}{ Demographic variables } & $\begin{array}{c}\text { Group I (Airtraq }{ }^{\circledR)} \\
(\mathbf{n = 3 0})\end{array}$ & Group II (McGrath®) $(\mathbf{n = 3 0})$ & P value \\
\hline Age (yr) & $42.30( \pm 15.51)$ & $41.97( \pm 14.68)$ & 0.852 \\
Gender (M:F) & $16: 14$ & $15: 15$ & 0.45 \\
Height (cm) & $155.36( \pm 6.42)$ & $153.55( \pm 6.39)$ & 0.577 \\
Weight (kg) & $55.17( \pm 6.95)$ & $54.75( \pm 6.79)$ & 0.754 \\
ASA (I:II) & $17: 13$ & $19: 11$ & 1 \\
Mallampati class (I:II:III) & $13: 8: 9$ & $11: 7: 12$ & 0.9 \\
MO (cm) & $4.25( \pm 0.203)$ & $4.27( \pm 0.205)$ & 0.588 \\
TMD (cm) & $7.38( \pm 0.209)$ & $7.37( \pm 0.205)$ & 0.547 \\
NC(cm) & $32.69( \pm 3.05)$ & $34.25( \pm 2.89)$ & 0.422 \\
\hline
\end{tabular}

Optimization manoeuvres to improve the glottis exposure were required in only 1 patient $(3.3 \%)$ in the Airtraq group versus $53.3 \%$ of patients (16 patients) in the McGrath group $\mathrm{P}<0.001$ [Table 2].

A significantly better Cormack lehane view was obtained with the Airtraq. It was of grade I in $73.3 \%$ (22 patients) with the Airtraq group versus 50\% (15 patients) in the McGrath group, $\mathrm{p}<0.001$ [Table 2].
The IDS scores were significantly lower in patients intubated with the Airtraq ${ }^{\circledR}$ compared with those intubated with either the McGrath laryngoscope $\mathrm{p}<0.001$. The total duration of endotracheal intubation was shorter in the Airtraq groupI $(19.18( \pm 2.86))$ seconds as compared to the McGrath group (28.27 $( \pm 5.09))$ seconds).

Table 2: Tracheal intubation data

\begin{tabular}{|l|c|c|}
\hline \multicolumn{1}{|c|}{ Intubation Parameter } & Group 1: Airtraq ${ }^{()} \mathbf{n = 3 0}$ & Group 2: McGrath n=30 $^{*}$ : \\
\hline Duration of tracheal intubation in & $19.18( \pm 2.86))$ & $28.27( \pm 5.09)^{*}$ \\
seconds & & \\
$\mathrm{n}(\%)$ & $22(73.3)$ & $15(50)^{*}$ \\
1 & $8(26.7)$ & $11(36.7)$ \\
2 & 0 & $4(13.3)$ \\
3 & $1(0,1)$ & $2(1,2)^{*}$ \\
Median (IQR) & & \\
No. of optimization manoeuvres (\%) & $29(96.7)$ & $14(46.7)^{*}$ \\
0 & $1(3.3)$ & $9(30)$ \\
1 & 0 & $7(23.3)$ \\
$\geq 2$ & $1(0,1)$ & $1(0,2)^{*}$ \\
Median (IQR) & & \\
Number of intubation attempts (\%) & & $20(66.7)$ \\
1 & $25(83.3)$ & $7(23.3)$ \\
2 & $4(13.3)$ & $3(10)$ \\
3 & $1(3.3)$ & $1(1,2)$ \\
Median(IQR) & $1(1,1)$ & $11(36.7)^{*}$ \\
IDS n (\%) & & $8(26.7)$ \\
Easy & $22(73.3)$ & $11(36.7)$ \\
Slight difficulty & $6(20)$ & $0(0)$ \\
Moderate to major Difficulty & $2(6.7)$ & \\
Impossible intubation & $0(0)$ & $2.5(1.1,3.8)^{*}$ \\
VAS difficulty score & & \\
Median (IQR) & $1(0.27,2.5)$ & \\
\hline
\end{tabular}

There were no cases of intubation failure in both the groups. VAS difficulty scores were significantly lower with the Airtraq $^{\circledR}$. The postoperative complications were comparable in both the groups [Table 3]. There was no incidence of oxygen desaturations, dental or other airway trauma with either of the video laryngoscopes. 
Table 3: Complications frequency with McGrath and Airtraq laryngoscopes

\begin{tabular}{|l|c|c|c|}
\hline Complications & $\begin{array}{c}\text { Group 1: } \\
\text { Airtraq }^{\circledR)} \mathrm{n}=30\end{array}$ & $\begin{array}{c}\text { Group 2: McGrath } \\
\mathbf{n = 3 0}\end{array}$ & P value \\
\hline Hypoxemia, SpO2 <92\% & 0 & 0 & \\
\hline Oral epithelium trauma & $2(6.67)$ & $1(3.3)$ & 0.65 \\
\hline Dental trauma & 0 & 0 & \\
\hline Blood-stained laryngoscope & $3(10)$ & $2(6.67)$ & 0.76 \\
\hline
\end{tabular}

Hemodynamic were measured preinduction and post intubation for up to 5minutes. There was no statistically significant difference between the two groups with respect to hemodynamic. [Table 4]

Table 4: Hemodynamic profile. Data are reported as mean (SD)

\begin{tabular}{|l|c|c|}
\hline Heart rate (beats/min) & Group 1: Airtraq $^{\circledR)} \mathbf{n = 3 0}$ & $\begin{array}{c}\text { Group 2: McGrath } \\
\mathbf{n = 3 0}\end{array}$ \\
\hline Pre-Induction & $78.52(14.11)$ & $75.66(13.04)$ \\
\hline Post-Intubation 1 min & $78.34(14.10)$ & $79.44(18.42)$ \\
\hline $2 \mathrm{~min}$ & $78.56(13.32)$ & $76.42(19.12)$ \\
\hline $3 \mathrm{~min}$ & $79.53(14.43)$ & $75.31(17.21)$ \\
\hline $5 \mathrm{~min}$ & $76.22(16.01)$ & $71.30(17.55)$ \\
\hline
\end{tabular}

\begin{tabular}{|c|c|c|}
\hline Mean arterial pressure $(\mathrm{mm}$ of $\mathrm{Hg})$ & 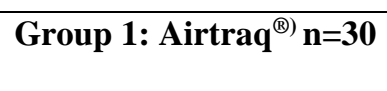 & $\begin{array}{c}\text { Group 2: McGrath } \\
n=30\end{array}$ \\
\hline Pre-Induction & $102.42(12.21)$ & $104.54(13.61)$ \\
\hline Post-Intubation $1 \mathrm{~min}$ & $84.77(20.36)$ & $88.82(22.43)$ \\
\hline $2 \min$ & $86.75(18.06)$ & $84.45(17.74)$ \\
\hline $3 \mathrm{~min}$ & $88.32(14.21)$ & $82.27(13.56)$ \\
\hline $5 \min$ & $78.51(14.35)$ & $76.33(14.52)$ \\
\hline
\end{tabular}

\section{Discussion}

Video laryngoscopes can be classified according to the presence of a guiding channel. In this study, we compared a video laryngoscope with a guiding channel (Airtraq) and one without a guiding channel (McGrath) for intubation by experienced anaesthesiologists in the normal airway scenario. The major factors which determine the success of endotracheal intubation include, the adequacy of laryngeal view obtained at laryngoscopy and the ease of manoeuvrability of the ETT into the glottis after its visualization. Although many studies have shown that video laryngoscopes offer better views of the glottis than conventional direct laryngoscopy, ${ }^{10}$ this was the only other study ${ }^{11}$ comparing the Airtraq with the McGrath in patients with normal airways, rather than on manikins.

The intubation difficulty score in the Airtraq was significantly better when compared to the McGrath. The number of optimization manoeuvres required in McGrath was also high. Both of these are due to the presence of channel in Airtraq which enables optimal positioning of the tube obviating the need for manoeuvring the ETT after visualizing the glottis. The ETT gets required curvature and proper alignment with respect to the visualizing lens in Airtraq, thus providing proper hand- eye coordination requested in indirect video laryngoscope. ${ }^{12,13}$

The laryngeal view as assessed by Cormack lehane grading was better in Airtraq compared to McGrath.
The superiority of the Airtraq ${ }^{\circledR}$ over McGrath for intubation is a probably due to its steeper curved blade which could better align the direction of its line of vision with the laryngeal axis. ${ }^{14}$ Our study results are in agreement with similar findings by another author. ${ }^{15}$ We used a modified Cormack-Lehane scoring system described by Yentis and Lee to compare the degree of glottis exposure. ${ }^{16}$ This classification of the glottis view, divides the traditional grade 2 into grade IIa (partial view of the glottis) and grade IIb (arytenoids or posterior parts of the cords just visible). This classification is advantageous as it identifies subtle degrees of decreased glottis exposure, (grade IIb), which may be translated into a potential difficult intubation.

Although visualization was similar in both the devices, time taken for intubation was significantly higher with McGrath than compared to Airtraq. This is because of the manoeuvring required for alignment of ETT into indirectly visualized glottis aperture, which requires proper hand- eye coordination in McGrath. Most of the patients in McGrath group therefore required bouggie to manoeuvre the ETT, hence requiring more time to provide the bouggie, and railroad the endotracheal tube.

With McGrath in our study, intubation failures were often because of problems with tube advancement, though the glottis view was good, demonstrating that tube advancement could be a problem with an 
unchannelled video laryngoscope like McGrath. Hence while using such video laryngoscopes it is preferable to use stillet or bouggie to avoid intubation failures and increase in laryngoscopy time.

We found a similar hemodynamic response to laryngoscopy with both the devices. In general the video laryngoscopes provide glottis view without a need to align the oro-pharyngeal-laryngeal axes and hence require less force to be applied during laryngoscopy. This translates into a lesser intubation response with these devices as compared to conventional direct laryngoscopes. Analysis of the VAS score revealed that the participating anaesthesiologists clearly expressed their overall preference for the Airtraq

We observed a similar incidence of airway and dental trauma with both Airtraq and McGrath, consistent with previous studies using these laryngoscopes. ${ }^{14,17} \mathrm{~A}$ greater force is required to align the glottis, pharyngeal, and laryngeal axes, in conventional laryngoscopy as compared to indirect laryngoscopes, which might explain the above. ${ }^{18}$ However some studies have observed a higher dental trauma score with McGrath as compared to the Airtraq ${ }^{\circledR}{ }^{19}$ This could be explained by the fact the McGrath ${ }^{\circledR}$ needs to lever back excessively to align the line of vision with the laryngeal axis, which leads to the dental trauma.

The strength of the study was that the study was prospective, randomized, well powered and performed in patients in the clinical setting, rather than on manikins.

Our study has some limitations. First, it was not possible to blind the anaesthesiologist to the laryngoscope being used. Hence double blinding could not be achieved, leading to some element of bias. Secondly, some parameters used in this study, such as VAS score, were subjective in nature. However, we found a good agreement between the subjective VAS and the more objective measures, such as the duration and success of tracheal intubation.

Our findings demonstrate the superiority of the channelled laryngoscope Airtraq compared to the McGrath in the clinical setting. In comparison with the McGrath laryngoscope, Airtraq reduced the time taken for tracheal intubation; the number of optimization manoeuvres required, improved the glottis view and increased the success rate of intubation. However, there were no differences among these devices regarding the hemodynamic responses to laryngoscopy. Further studies are necessary to confirm if these findings extend to patients with difficult airway as well.

\section{Conflicts of Interest: Nil}

\section{References}

1. Frerk C, Difficult Airway Society intubation guidelines working g, Mitchell VS, Difficult Airway Society intubation guidelines working g, McNarry AF, Difficult Airway Society intubation guidelines working g, et al. Difficult Airway Society 2015 guidelines for management of unanticipated difficult intubation in adults. BJA: Br J Anaesth. 2015;115(6):827-848.

2. Stroumpoulis K, Pagoulatou A, Violari M, Ikonomou I, Kalantzi N, Kastrinaki K, et al. Videolaryngoscopy in the management of the difficult airway: a comparison with the Macintosh blade. Eur J Anaesth. 2009;26(3):218-22. PubMed PMID: 19237983. Epub 2009/02/25. eng.

3. Lewis SR, Butler AR, Parker J, Cook TM, Smith AF. Videolaryngoscopy versus direct laryngoscopy for adult patients requiring tracheal intubation. The Cochrane database of systematic reviews. 2016;11:CD011136. PubMed PMID: 27844477. Epub 2016/11/16. eng.

4. Shippey B, Ray D, McKeown D. Case series: the McGrath videolaryngoscope--an initial clinical evaluation. Canadian journal of anaesthesia $=\mathrm{J}$ Can Anesth. 2007;54(4):307-313. PubMed PMID: 17400984. Epub 2007/04/03. eng.

5. Lu Y, Jiang H, Zhu YS. Airtraq laryngoscope versus conventional Macintosh laryngoscope: a systematic review and meta-analysis. Anaesthesia.

2011;66(12):1160-1167. PubMed PMID: 21883130. Epub 2011/09/03. eng.

6. Shin M, Bai SJ, Lee K-Y, Oh E, Kim HJ. Comparing McGRATH $®$ MAC, C-MAC® and Macintosh Laryngoscopes Operated by Medical Students: A Randomized, Crossover, Manikin Study. Biomed Res Int. 2016;2016:2018.

7. Adnet MDPF, Borron MDMSStephen W, Racine MDStephane X, Clemessy MDJ-L, Fournier MDJ-L, Plaisance MDP, et al. The Intubation Difficulty Scale (IDS) Proposal and Evaluation of a New Score Characterizing the Complexity of Endotracheal Intubation. Anesthesiol. 1997;87(6):1290-1297.

8. Krage R, van Rijn C, van Groeningen D, Loer SA, Schwarte LA, Schober P. Cormack-Lehane classification revisited. BJA: Br J of Anaesth. 2010;105(2):220-227.

9. Maharaj CH, Buckley E, Harte BH, Laffey JG. Endotracheal intubation in patients with cervical spine immobilization: a comparison of macintosh and airtraq laryngoscopes. Anesthesiol. 2007;107(1):53-59. PubMed PMID: 17585215. Epub 2007/06/23. eng.

10. Su YC, Chen CC, Lee YK, Lee JY, Lin KJ. Comparison of video laryngoscopes with direct laryngoscopy for tracheal intubation: a meta-analysis of randomised trials. Eur J Anaesthesiol. 2011;28(11):788-795. PubMed PMID: 21897263. Epub 2011/09/08. eng.

11. Raza N, Hasan M, Ahmed S, Bano S, Athar M. A comparative study of McGrath and Airtraq videolaryngoscopes for tracheal intubation. $\mathrm{J}$ Anaesthesiol Clin Pharmacol. 2017;33(2):221-225.

12. Di Marco P, Scattoni L, Spinoglio A, Luzi M, Canneti A, Pietropaoli P, et al. Learning curves of the Airtraq and the Macintosh laryngoscopes for tracheal intubation by novice laryngoscopists: a clinical study. Anesth and Analgesia. 2011;112(1):122-125. PubMed PMID: 21048093. Epub 2010/11/05. eng.

13. Giquello JA, Humbert S, Duc F, Monrigal JP, Granry JC. [Use of the Airtraq by inexperienced physicians supervised during a series of tracheal intubation in adult patient with anticipated difficult airway]. Annales francaises d'anesthesie et de reanimation. 2011;30(11):804-808. PubMed PMID: 21719241. Epub 2011/07/02. Utilisation de l'Airtraq par des praticiens inexperimentes supervises pour une serie de patients adultes prevus difficiles a intuber. fre. 
14. Kaki A, AlMarakbi W, Fawzi H, Boker A. Use of Airtraq, C-Mac, and Glidescope laryngoscope is better than Macintosh in novice medical students' hands: A manikin study. Saudi J Anaesth. 2011;5(4):376-381.

15. Savoldelli GL, Schiffer E, Abegg C, Baeriswyl V, Clergue F, Waeber JL. Comparison of the Glidescope, the McGrath, the Airtraq and the Macintosh laryngoscopes in simulated difficult airways*. Anaesth. 2008;63(12):13581364. PubMed PMID: 19032306. Epub 2008/11/27. eng.

16. Yentis SM, Lee DJ. Evaluation of an improved scoring system for the grading of direct laryngoscopy. Anaesth. 1998;53(11):1041-4. PubMed PMID: 10023271. Epub 1999/02/19. eng.

17. Maharaj CH, Higgins BD, Harte BH, Laffey JG. Evaluation of intubation using the Airtraq ${ }^{\circledR}$ or Macintosh laryngoscope by anaesthetists in easy and simulated difficult laryngoscopy - a manikin study*. Anaesth. 2006;61(5):469-477.

18. Niforopoulou P, Pantazopoulos I, Demestiha T, Koudouna E, Xanthos T. Video-laryngoscopes in the adult airway management: a topical review of the literature. Acta anaesthesiologica Scandinavica.
2010;54(9):1050-61. PubMed PMID: 20887406. Epub 2010/10/05. eng.

19. Owada G, Mihara T, Inagawa G, Asakura A, Goto T, Ka $K$. A comparison of the Airtraq(R), McGrath(R), and Macintosh laryngoscopes for difficult paediatric intubation: A manikin study. PloS one. 2017;12(2):e0171889. PubMed PMID: 28187213. Pubmed Central PMCID: PMC5302788. Epub 2017/02/12. eng.

How to cite this article: Padhy S, Jayaram K, Sindhuja, Priyanka. Comparison of channelled vs unchannelled video laryngoscope- A prospective randomised trial. Indian $\mathbf{J}$ Clin Anaesth. 2018;5(4):543-548. 